\title{
Rezension zu Röhner, Charlotte/Hövelbrinks, Britta (eds.) (2013): Fachbezogene Sprachförderung in Deutsch als Zweitsprache. Theoretische Konzepte und empirische Befunde zum Erwerb bildungssprachlicher Kompetenzen. Weinheim, Basel: Beltz
} Juventa.

\author{
Esin Işil Gülbeyaz (Potsdam)
}

Der von Röhner und Hövelbrinks herausgegebene Sammelband widmet sich mit dem Titel "Fachbezogene Sprachförderung in Deutsch als Zweitsprache" einem der aktuellsten Themen des Faches "Deutsch als Zweitsprache". Einen besonderen Stellenwert räume ich der Veröffentlichung ein, weil Röhner und Hövelbrinks auf die Barrieren und mangelhafte Unterstützung mehrsprachiger ${ }^{1}$ SchülerInnen ${ }^{2}$ beim Erwerb der Zweit- bzw. Bildungssprache im deutschen Bildungssystem aufmerksam machen. Einige dieser Mängel und Barrieren benennt Röhner in der Einleitung wie folgt:

- Konzentration der bildungspolitischen Konzepte auf den Ausbau vorschulischer Bildung und Sprachförderung sowie Nichtberücksichtigung der schulstrukturellen Bedingungen als Ursache der Bildungsungleichheit;

- Drei grundlegende Barrieren (nach Tajmel 2010) im Bildungssystem für mehrsprachige Kinder und Jugendliche: (1) Sprachliche Barrieren, bedingt durch mangelnde Berücksichtigung des Sprachstandes der Schüler; (2) Kulturelle Barrieren, bedingt durch die "Erwartung und Bevorzugung eines bestimmten kulturellen Habitus" (Bordieu 1992, Gogolin 1994, zitiert nach Röhner und Hövelbrinks S. 7); (3) Institutionelle Barrieren, bedingt durch das selektive Schulsystem;

- Fokus der Bildungspolitik - entgegen den sprachpädagogischen Grundsätzen und Empfehlungen der KMK von 2002 zur Mehrsprachigkeit als Ziel - auf die einseitige Förderung der Verkehrssprache Deutsch;

- Betrachtung natürlicher, individueller Mehrsprachigkeit der Migrantenkinder als Bildungshindernis, anstatt diese $\mathrm{zu}$ fördern, und Erwartung einer "einseitigen sprachlichen und kulturell-sozialen Integration und Anpassung".

Folglich positioniert sich der Band gegen "eine sprachliche Priorisierung des Deutschen" und vereint Beiträge zu einem sprachsensiblen Fachunterricht für Deutsch als Zweitsprache zu einer Didaktik der Sprachenvielfalt. Daher schließt der Sammelband von Röhner und

\footnotetext{
1 Terminologiewahl der Rezensentin.

Linguistik online 67, 5/14 - http://dx.doi.org/10.13092/lo.67.1604

licensed under CC 3.0
} 
Hövelbrinks mit seinem Fokus auf integrative Ansätze zur fachbezogenen Sprachförderung eine wichtige Forschungslücke. Ferner trägt der Band der Idee des Europäischen Kerncurriculums für eine inklusive Förderung der Bildungssprache (Inclusive Academic Language Teaching - ECC IALT) Rechnung.

Der Band ist in vier Teile gegliedert. Der erste Teil geht auf didaktische Konzepte der fachbezogenen Sprachförderung sowie den Forschungsstand ein. Der das Kapitel eröffnende Artikel Röschs ("Integrative Sprachbildung im Bereich Deutsch als Zweitsprache") gibt zunächst einen Überblick über integrative Ansätze und empirische Befunde zur Sprachförderung. Dabei wird betont, dass zur Förderung des Sprachlernens/-lehrens im Fachunterricht konsequenterweise bei der Aus- und Fortbildung des pädagogischen Personals anzusetzen ist, wie z. B. durch "eine linguistische und sprachdidaktische Grundbildung in allen Bildungsfächern" (S. 34). Selten und daher wichtig ist ferner die terminologische Differenzierung Röschs, Schüler mit Deutsch als Zweitsprache nicht als Synonym für solche mit Migrationshintergrund $\mathrm{zu}$ verwenden. Im darauf folgenden Artikel erläutern Beese und Benholz ("Sprachförderung im Fachunterricht. Voraussetzungen, Konzepte und empirische Befunde") integrative Konzepte zur Koordinierung von Sprachkompetenzen und Fachinhalten wie Scaffolding, SIOP (Sheltered Instruction Observation Protocol) und CLIL (Content and Language Integrated Learning). Die Autorinnen gehen zudem auf die empirische Unterrichtsforschung ein und erwähnen wichtige Konsequenzen für Forschung und Lehrerbildung. Der letzteren weisen sie eine Schlüsselrolle bei der Realisierung und Weiterentwicklung fachlicher Sprachförderung zu.

Der zweite Teil befasst sich mit der Rolle der Sprache im Fachunterricht. In dem ersten Artikel dieses Kapitels nennt Grießhaber ("Die Rolle der Sprache bei der Vermittlung fachlicher Inhalte") als ein besonderes Merkmal der fachsprachlichen Kommunikation die fachspachliche Lexik, für welche Substantive und insbesondere Komposita besonders charakteristisch seien. Anschließend schildert er anhand zweier exemplarischer Untersuchungen die Schwierigkeiten, die sich im Bereich der Lexik vor allem für DaZLernende ergeben. Die Untersuchung eines Sachunterrichtslehrwerks der 3. Grundschulklasse sowie die Studie zur mündlichen Kommunikation im mathematischen Anfangsunterricht zeigen, dass zum einen Begriffserklärungen zu kurz kommen, aber auch syntaktische Strukturen für Schüler fordernd sein können. Andererseits wird beobachtet, dass die Probanden mit DaZ Wortschatzlücken und Schwierigkeiten bei der Verschriftlichung mündlich prozessierten Wissens aufweisen. Vor dem Hintergrund dieser Ergebnisse stellt Grießhaber zwei relevante Fragen: zum einen nach der Verfügbarkeit des im Unterrichts vermittelten fachlichen Wissens überhaupt, zum anderen nach der "Tragfähigkeit der auf muttersprachlich deutsche Schüler ausgerichteten Vermittlungsverfahren".

Die Beschäftigung mit der Bedeutung der Bildungssprache wird durch Hövelbrinks ("Die Bedeutung der Bildungssprache für Zweitsprachlernende im naturwissenschaftlichen Anfangsunterricht") fortgesetzt, die auf die Bildungssprache unter Berücksichtigung des naturwissenschaftlichen Anfangsunterrichts eingeht. Sie konstatiert durch eine eigene Studie, dass bildungssprachliche Kompetenzen sich bei DaZ-Lernenden - unabhängig vom

\footnotetext{
${ }^{2}$ Aus Gründen der besseren Lesbarkeit wird auf die gleichzeitige Verwendung männlicher und weiblicher Sprachformen verzichtet.
} 
Vorhandensein eines "Förderbedarfs" - im 1. Schuljahr beobachten lassen. In der Folge gelte es zu untersuchen:

- welche Merkmale das Register der Bildungssprache auszeichnen;

- ob und wie diese Merkmale für Zweitsprachlernende wirken könnten;

- wie sich die zu Schulanfang vorhandenen bildungssprachlichen Kompetenzen auf das fachliche Lernen auswirken und dahingehend gestärkt werden können;

- wie bildungssprachliche Erwerbsprozesse und damit einhergehend die Operationalisierung für diagnostische Instrumente aussehen.

Ahrenholz ("Sprache im Fachunterricht untersuchen") skizziert in seinem Beitrag das Projekt "Fachunterricht und Deutsch als Zweitsprache" (Fach-DaZ), das Erkenntnisse in den Bereichen Satzlänge, Nebensätze und Verben zu gewinnen bezweckt, "um die sprachlichen Mittel zu beschreiben, die bei der Wissensvermittlung und Wissensaneignung in verschiedenen Fächern und verschiedenen Altersgruppen von Bedeutung sind" (S. 96) und von Schülern verlangt werden. Es werden hier vorläufige Befunde zu Schulbüchern und Fachunterricht am Beispiel von Biologiebüchern und Biologieunterricht dargestellt. Das Projekt füllt insofern eine Forschungslücke, welche auch von Ahrenholz u. a. erwähnt wird, als sprachliche Mittel in Fachlehrwerken und im Fachunterricht erforscht und mögliche Schwierigkeiten der Schüler auf empirischer Grundlage eruiert werden.

Peschel ("Schreiben in der Zweitsprache Deutsch - Orientierung an Textmustern im Fachunterricht") befasst sich mit der textmusterorientierten Sprachförderung. Sie erklärt zunächst die Termini Textsorte, Textmuster und Textmusterwissen und geht im nächsten Schritt auf Grundfragen und Forschungsergebnisse ein. Bei didaktischen Vorschlägen zum Schreiben in der Zweitsprache stellt sie zwei fest verankerte schreibdidaktische Ansätze dar: freies und kreatives Schreiben mit seiner motivierenden Wirkung vs. generatives Schreiben, dem eine Vorbildfunktion zugesprochen wird. Als "eine gelungene Brücke zwischen diesen beiden Positionen" (S. 105) bezeichnet sie als weiteren sprachdidaktischen Ansatz das 3Phasen-Modell von Schmölzer-Eibinger (2008), in welchem reproduktive sowie produktive Aspekte verbunden würden. Die Wirksamkeit des letzten Ansatzes wird anhand der Analyse eines Beispieltextes (in Bezug auf Orthografie, Grammatik, Lexik und Textbau) illustriert, der ein Versuchsprotokoll aus dem Chemieunterricht darstellt, verfasst von einer TürkischDeutsch mehrsprachigen Schülerin.

Der dritte Teil des Sammelbandes widmet sich der Förderung bildungs- und schriftsprachlicher Kompetenzen vom Kindergarten bis zur Hochschule. Diesen Teil eröffnen Hopf und Eckhardt ("Bildungssprache im Kindergarten. Ein Blick in die konzeptionellen Umsetzungen der Rahmenvorgaben für die frühe Bildung") mit ihrem Beitrag zur Bildungssprache im Kindergarten. Sie untersuchen exemplarisch Bildungs- und Erziehungspläne und zeigen auf, inwiefern bildungssprachliche Fähigkeiten in diesen berücksichtigt werden. Ferner befassen sie sich mit der Frage nach dem Anforderungsprofil pädagogischer Lehrkräfte in Bezug auf Aspekte der Bildungssprache. Hopf und Eckhardt stellen fest, dass sowohl im Nationalen Integrationsplan als auch in den Bildungs- und Erziehungsplänen theoretische Bezüge zur bildungssprachlichen Förderung sich zwar finden, 
jedoch die Umsetzung einer integrierten Sprachförderung lediglich rudimentär behandelt wird.

Sprachförderung im Grundschulbereich wird beispielhaft von Babbe ("'Du hast dich mit einer Jacke warm gemacht?' - Sprachförderung in der Erika-Mann-Grundschule") behandelt. Sie stellt in ihrem Beitrag das Sprachkonzept der Erika-Mann-Schule in Berlin vor, das die Schule, deren Schülerschaft zu $82 \%$ aus Kindern mit Migrationshintergrund besteht, eigens für eine durchgängige Sprachbildung nach Gogolin und Lange (2011) entworfen hat. Die zwei Hauptkomponenten des Sprachkonzepts beziehen sich auf Language Awareness von James und Garrett (1992) und auf Academic Language von Cummins (2008). Babbe berichtet, dass die Schule dank ihrem Sprachkonzept eine deutliche Zunahme an Anmeldungen zum Schulbesuch aus bildungsorientierteren Familien verzeichnet.

Gierth ("Textüberarbeitung von Jugendlichen mit türkischem Migrationshintergrund in der Sekundarstufe I") skizziert in ihrem Beitrag eine explorative Studie zur Textüberarbeitung von Jugendlichen mit türkischem Migrationshintergrund in der Sekundarstufe I und zeigt anhand zweier Fallanalysen Tendenzen auf. Gegenübergestellt werden die Textversionen einer Seiteneinsteigerin mit einer Bildungsinländerin, die beide die achte Klasse einer Hauptschule besuchen und Türkisch als Erst- bzw. Familiensprache haben. Die in der Türkei geborene Schülerin weist dabei bzgl. der inhaltlichen Richtigkeit eine höhere Textüberarbeitungskompetenz auf als die in Deutschland geborene und beschulte Schülerin, die jedoch bei der Orthographie und Interpunktion bessere Leistungen erbringt. Dies wäre nicht verwunderlich, wenn man annehmen könnte, dass die in Deutschland geborene Schülerin nur in der deutschen Sprache alphabetisiert und nur an das deutsche Schriftsystem herangeführt wurde, wohingegen die Seiteneinsteigerin sich nach dem türkischen Schriftsystem das deutsche zusätzlich aneignen muss.

Siebert-Ott und Decker ("Entwicklung und Förderung akademischer Text- und Diskurskompetenz in der Zweitsprache Deutsch zu Studienbeginn") berichten in ihrem Beitrag über ein Teilprojekt im Rahmen des Verbundprojektes zur Modellierung, Messung und Förderung der Textkompetenzen von Lehramtsstudierenden. Das Teilprojekt hat "die Entwicklung von Aufgabenformaten zur Integration von fachlichem und sprachlichem Lernen im Lehramtsstudium" zum Ziel. Ein besonderer Fokus liegt dabei auf der Entwicklung sprachlicher Kompetenzen von Studierenden aus Familien mit Zuwanderungsgeschichte. Anhand eines im Rahmen des Projektes entwickelten Kriterienkatalogs werden die von den Studierenden eingereichten Arbeiten bewertet. Die Feedbackbögen enthalten sowohl inhaltliche als auch sprachliche Kriterien. Die Modellierung, Beurteilung und Förderung akademischer Textkompetenz erfolgt anhand eines dreidimensionalen Kompetenzmodells. Im Beitrag werden ferner "Anforderungen an gut profilierte Schreibaufgaben" genannt. Dargestellt werden zudem empirische Daten $\mathrm{zu}$ einführenden fachdidaktischen Lehrveranstaltungen sowie Schreibaufgaben. In diesem Zusammenhang wird auf Diskrepanzbefunde aus anderen Quellen hingewiesen, auf Diskrepanzbefunde sowohl innerhalb einer Institution (beim Übergang von der Sekundarstufe I zum Gymnasium) als auch zwischen den Institutionen (beim Übergang vom Gymnasium zur Hochschule). Diesem Beitrag kommt insofern eine besondere Rolle zu, als die Autorinnen die Bedeutung des "Lesens und Schreibens in Prozessen der Wissensaneignung und der Wissensprüfung im Studium" (S. 169) unterstreichen und folglich dafür plädieren, dass Lehrveranstaltungen am 
Beginn des Studiums dahingehend konzipiert werden sollten, dass "die Kompetenzerwartungen für die Studierenden transparent gemacht werden und dass die Kompetenzentwicklung der Studierenden mit gut profilierten Lese- und Schreibaufgaben systematisch unterstütz wird" (S. 169).

Der letzte und vierte Teil geht dem Thema zum integrierten Sprach- und Fachlernen als Aufgabe der Lehrerprofessionalisierung nach. Zunächst geben Pitton und Scholten-Akoun ("Deutsch als Zweitsprache als verpflichtender Bestandteil der Lehramtsausbildung in Nordrhein-Westfallen - eine vorläufige Bestandsaufnahme") einen Überblick über das DaZModul als verpflichtenden Bestandteil der Lehramtsausbildung an den Universitäten Nordrhein-Westfallens. Mit der Einführung des modularisierten Lehrerausbildungssystems zum Wintersemester 2012/2013 wurde der DaZ-Bereich zum obligatorischen Bestandteil der Lehrerausbildung, für welchen im Lehrerausbildungsgesetz mindestens 6 Leistungspunkte vorgesehen sind. Die Bestandsaufnahme Pittons und Scholten-Akouns zeigt, dass alle nordrhein-westfälischen Universitäten ihre DaZ-Module in diesem Mindestumfang gestalten, mit Ausnahme der Universität Duisburg-Essen, die das DaZ-Mercator-Modul als integralen Bestandteil der Lehrerausbildung eingeführt hat, welches mit 12 Leistungspunkten den doppelten Mindestumfang vorsieht. Aufgrund der zusätzlichen, fächerspezifischen, fakultativen DaZ-Anteile, umgesetzt in Zusammenarbeit mit Fachdidaktiken, kommt der Universität Duisburg-Essen und dem DaZ-Mercator-Modul dabei eine besondere Bedeutung zu. Dass der Erfolg der DaZ-Module und -Veranstaltungen von der personellen Ausstattung abhängt, bleibt nicht unerwähnt. Die Frage, inwieweit alle Lerninhalte des DaZ-Moduls in 6 Leistungspunkten vermittelt werden können, steht allerdings offen.

Tajmel ("Möglichkeiten der sprachlichen Sensibilisierung von Lehrkräften naturwissenschaftlicher Fächer") schlägt fünf Möglichkeiten der sprachlichen Sensibilisierung für mathematisch-naturwissenschaftliche Lehrkräfte vor. Diese sind kontrastive Sprachbetrachtung, Wechsel von der Lehrerperspektive zur Schülerperspektive, Identifikation von Sprachhandlungen im Fach, differenzierte Beurteilung von Schülertexten, Formulierung sprachlicher Erwartungen und sprachlicher Lernziele. Tajmel betont in ihrem Beitrag nicht nur die Relevanz am Fach orientierter sprachlicher Sensibilisierung der Lehrkräfte als Grundlage für eine Professionalisierung im Bereich der fachbezogenen Sprachförderung, sondern sie gibt mit den fünf Möglichkeiten nützliche Handreichungen an (angehende) Lehrkräfte.

Den vierten Teil und somit den Sammelband schließen Thürmann und Vollmer ("Schulsprache und Sprachsensibler Fachunterricht: Eine Checkliste mit Erläuterungen"). Die Autoren stellen eine Checkliste vor, die sie zur Entwicklung eines "sprachbewussten bzw. sprachsensiblen" Fachunterrichts entwarfen. Sie soll Fachlehrkräften bei der Identifizierung der Sprachsensibilität des Fachunterrichts sowie bei der (kritischen) Reflektion über diese Sprachsensibilität als Handreichung dienen. Anzumerken ist, dass der Gebrauch des Begriffes "Risikoschüler" trotz des Hinweises auf das andere Verständnis des Wortes im Sinne der PISA unglücklich ist, denn durch den Hinweis wird die implizite Schuldzuweisung zum Schüler für seine zu verbessernden Leistungen nicht getilgt.

Insgesamt liegt mit dem Sammelband von Röhner und Hövelbrinks eine Veröffentlichung vor, die nicht nur auf die Bedeutung des in der Praxis noch defizitiären sprachsensiblen 
Unterricht(en)s hinweist, sondern auch für verschiedene Lernbereiche und Jahrgänge beispielhafte Handreichungen aus den dargestellten Projekten gibt. Die im Sammelband dargestellten Ansaetze eignen sich nicht nur zur Förderung mehrsprachiger Kinder, sondern ebenfalls zum sprachsensiblen Unterricht für alle Schüler in gemeinsamen Klassen. Ferner werden Forschungslücken sowie Desiderate genannt, weshalb ich die Veröffentlichung neben angehenden und praktizierenden Lehrern auch (forschenden) Hochschullehrern der relevanten Fachrichtungen empfehlen möchte. Ein allgemeines terminologisches Problem, das in deutschsprachigen Publikationen und Fachkreisen $\mathrm{zu}$ beobachten ist, macht sich auch in diesem Band bemerkbar.

Es werden für mehrsprachige Schüler äußerst unterschiedliche Begriffe genannt, die teilweise auf einen wie auch immer gearteten Migrationshintergrund verweisen (z. B.: Schülerinnen und Schüler bzw. Kinder und Jugendliche mit Migrationshintergrund; Schülerinnen und Schüler mit Zuwanderungsgeschichte) oder welche den inzwischen umstrittenen Begriff "Muttersprache" thematisieren (z. B.: Schülerinnen und Schüler, deren Muttersprache nicht Deutsch ist). Teilweise sind die Begriffe redudant (z. B.: Seiteneinsteigerin mit Migrationshintergrund) oder widersprüchlich $\quad$ (z. B. bildungsinländische Migrantenjugendliche) oder abwertend (z. B.: Risikoschüler).

Mit diesen Bezeichnungen wird die Gruppe derjenigen Kinder und Jugendlichen nicht abgedeckt, die zu Hause neben dem Deutschen eine andere Familiensprache (z. B. Türkisch oder Spanisch etc.) oder zwei verschiedene Familiensprachen (Spanisch-Türkisch, JapanischFranzösisch) sprechen und Deutsch - der Erwerbsfolge nach - als Drittsprache erlernen. Angesichts der Tatsache, dass die Anzahl solcher mehrsprachigen Kinder zunimmt, und aus dem Grunde der terminologischen Adäquatheit sowie auf Grund des Fokus des Faches DaZ auf dem sprachsensiblen Unterricht(en) entspricht meines Erachtens der Terminus "mehrsprachige Kinder und Jugendliche bzw. Schüler" sowohl der lebensweltlichen als auch gesellschaftlichen Realität. 